\title{
Rewarding and aversive effects of amygdaloid self-stimulation as a function of current intensity
}

\author{
Solomon S. Steiner and M. R. D'Amato
}

NEW YORK UNIVERSITY

\begin{abstract}
Thirsty rats chose between two bars, one producing water alone and the other, water plus electrical brain stimulation. With electrode emplacements in the amygdala, low current intensities were aversive while, paradoxically, higher intensities were rewarding.
\end{abstract}

\section{Problem}

Electrical intracranial self-stimulation (ICS) can act as (1) an exclusively positive reinforcer, (2) an exclusively negative reinforcer, or (3) as both a positive and negative reinforcer, being positive at relatively low current intensities and negative at higher amperages (Delgado, Roberts, and Miller, 1955; Olds, 1956; Reynolds, 1958). A fourth possibility, namely, that ICS can be converted from a negative to a positive reinforcer by an increase in stimulating current, has not yet been demonstrated, so far as we know. This report describes such a finding.

\section{Method}

One male and two female rats, 10 months old at the start of the study, served as Ss. They were implanted with bipolar electrodes in the amygdaloid region.

The apparatus consisted of a two-bar Skinner box, a square wave generator, and associated programming equipment. The current levels reported were obtained from oscilloscopic measurements.

In a preliminary experiment, thirsty rats were trained to press a bar that delivered both water and ICS. Low ICS intensity was clearly aversive, but higher currents ofter appeared, unexpectedly, to be rewarding. In order to evaluate this possibility, the three rats, still thirsty, were allowed to choose between two bars, one of which, when pressed, delivered water reward and the other, water plus . $25 \mathrm{sec}$. of ICS. During a single daily 20-min. session, ICS was maintained at a fixed intensity, but it was shifted from the right to the left bar every 2 min. (Ss had been trained previously to shift between the two bars to obtain water reward alone.) Each 2-min. period was scored plus if S pressed the water-plus-ICS bar more often than the water-alone bar. If, therefore, ICS intensity during a given session was aversive, the proportion of pluses should be small; if, on the other hand, it was rewarding, the proportion of pluses should be rather high. Since each $\mathrm{S}$ was tested at each intensity on two separate days, the total number of pluses could vary between 0 and 20 .

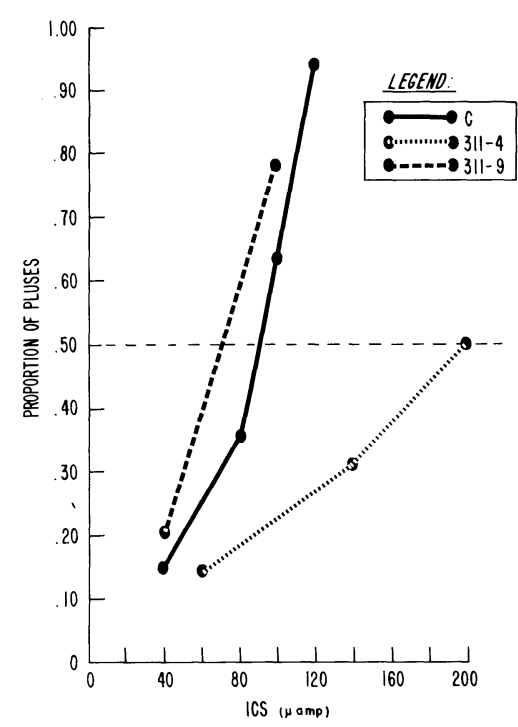

Fig. 1. Each point shows the proportion of $2 \mathrm{~min}$. sessions during which Spressed the bar delivering water plus ICS more often than the bar producing water alone.

\section{Results}

The choice data are graphed in Fig. 1. At low amperages, all three Ss clearly avoided the water-plus-ICS bar. Evaluated by a two-tailed sign test, the distribution of responses for each S departs from chance expectation (rat C, $40 \mu \mathrm{amp}$, $\mathrm{P}=.004$; rat $311-9,40 \mu \mathrm{amp}, \mathrm{P}=.012$; rat $311-4$, $60 \mu$ amp, $\mathrm{P}=.001)$. The low intensities, it therefore seems clear, were aversive.

At the higher intensities, on the other hand, two of the rats showed a marked preference for the water-plus-ICS bar (rat C, $120 \mu$ amp, P = .001 ; rat $311-9,100 \mu \mathrm{amp}, \mathrm{P}=.030)$. In the case of the third S (311-4), the highest intensity employed ( $200 \mu \mathrm{amp})$, while no longer aversive, does not seem to be rewarding. The 50-percent proportion, however, is accounted for by the fact that at this intensity the $\mathrm{S}$ developed a position habit of pressing only the right bar. During the periods when this bar was delivering water plus ICS, the rat rarely bothered to drink the water delivered. S therefore seemed to be pressing for the ICS alone. 


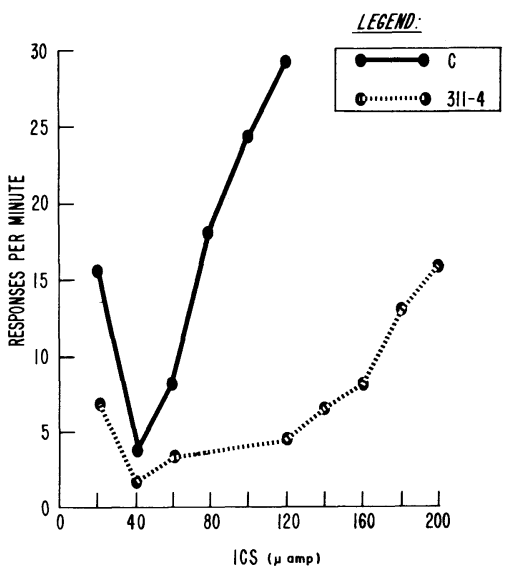

Fig. 2. Rate of bar pressing for ICS alone as a function of intensity.

To check the possibility that the reversal of ICS reinforcing value might depend on concurrent thirst and/or water reward, the data of Fig. 2 were obtained. Because of repeated convulsions, complete data could not be obtained for rat 311-9. Those for the other two rats, however, were collected in two $60-$ min. sessions while they were satiated for water and pressed a single bar for ICS alone. ICS intensity was changed every 10 min. The results are clearly consistent with the choice-response data. One implication of both Figs. 1 and 2 is that the reversal property of ICS may easily be missed if an investigator selects ICS amperages that maximize response rate, which is a common procedure.

Histological examination placed the electrodes of rat 311-4 in the ventral border of the central amygdaloid nucleus, lateral to the Stria terminalis and ventral and posterior to the head of the caudate nucleus (estimated DeGroot coordinates: A 5.0, L 4.4, D -1.6). The electrode tips of rat 311-9 were on the lateral border of the medial amygdaloid nucleus (A 5.0, L 4.3, D-2.8); those of rat $C$ in the ventral border of the medial amygdaloid nucleus (A 6.2, L 2.5, D -2.6), further anterior than the electrodes of the other animals.

Although all these placements were in the amygdaloid region, the shift from aversiveness to positiveness with increasing stimulation current can apparently occur with placements in other areas. Recently we obtained it in a rat with electrodes near the fornix (A 4.5, L 1.2, D - 3.1).

\section{Discussion}

As intensity of ICS is increased, current presumably spreads to points farther away from the electrode tips. On this assumption, the reversal from positive to negative previously reported for certain regions of the hypothalamus (Olds, 1956) could be explained as a spread from positively to negatively reinforcing structures. If this explanation is applied to our reversal in the other direction, we would have to assume (1) that the negatively reinforcing structures near our electrodes are considerably less extensive than neighboring structures or else much less "powerful" unit for unit, and (2) rewarding stimulation can eliminate the marked aversiveness evident at lower intensities. Another, to us more plausible, possibility is that the higher stimulating currents inhibit the activation of negatively reinforcing structures that occurs with low amperages (cf. Egger and Flynn, 1962).

\section{References}

DELGADO, J. M. R., ROBERTS, W. W., and MILLER, N. E. Learning motivated by electrical stimulation of the brain. Amer. J. Physiol., 1954, 179, 587-593.

EGGER, M. D., and FLYNN, J. P. Amygdaloid suppression of hypothalamically elicited attack behavior. Science, 1962, 136, 43-44.

OLDS, J. A preliminary mapping of electrical reinforcing effects in the rat brain.J. comp. physiol. Psychol., 1956, 49, 281-285.

REYNOLDS, R. W. The relationship between stimulation voltage and rate of hypothalamic self-stimulation in the rat. J.comp. physiol. Psychol., 1958, 51, 193-198.

\section{Acknowledgment}

This work was supported in part by Grant G-14724 from the National Science Foundation; it was completed during the first author's tenure of an NIH predoctoral fellowship. We thank Dr. D. Webster and Mr. E. Coons for independently determining the sites of the electrode placements. 